\title{
Mediterránea
}

\section{Roberto APARICI*}

Docente e investigador. Universidad Nacional de Educación a Distancia (UNED). raparici@edu.uned.es

La Cultura de la Participación

The culture of participation

\section{Sara OSUNA ACEDO*}

Docente e investigadora. Universidad Nacional de Educación a Distancia (UNED). sosuna@edu.uned.es

Fecha de recepción: 05/11/2013

Fecha de revisión: 26/11/2013

Fecha de preprint: 10/12/2013

Fecha de publicación final: 30/12/2013

\section{Resumen}

La cultura de la participación implica modelos de comunicación horizontales donde las relaciones de poder proporcionan a cada individuo la posibilidad de intercambiar puntos de vista, expresar ideas, comentarios, experiencias así como trabajar colaborativamente. Esta cultura de la participación implica pensar y desarrollar una cultura de la comunicación que supere los modelos heredados de las teorías funcionalistas. La Web 2.0, en general, y las redes sociales, en particular, son los escenarios adecuados para la participación que ponen en juego los principios de la inteligencia colectiva, la intercreatividad y la arquitectura de la participación. En este escenario de la Web 2.0 se construye un ecosistema infocomunicativo que modifica todas las reglas tradicionales sobre audiencias. ¿̇Qué tipo de público son los públicos de la participación? ¿̇Qué tipo de interacciones establecen entre sí? ¿ Cómo se participa y qué calidad de participación se establece?. La ciudadanía, para Manuel

\begin{abstract}
The culture of participation involves horizontal models of communication where relations of power give individuals the possibility to exchange points of view, express ideas, comments, and experiences as well as work together collaboratively. This culture of participation involves the thinking and development of a culture of communication that overcomes traditional models established in functionalist theories. Web 2.0 in general, and social networks, in particular, are suitable scenarios for participation that promote the principles of collective intelligence, interactivity, and an architecture of participation. In the scenario of Web 2.0 an info-communicative ecosystem is constructed which modifies all traditional rules of audiences. Which type of publics are publics of participation? What types of interactions are established among them? How do they participate and what quality of participation is established? Citizenship, according to Manuel Castells (1998), is a great interactive mind, or as in the terms of Pisani and Piotet (2009) a web-
\end{abstract}


Castells (1998) es una gran mente interactuante o una webactora en términos de Pisani y Piotet (2009) con capacidad para crear dentro del espacio digital su propia información y su propio estilo comunicativo. Esta ciudadanía digital participa en los escenarios virtuales dejando su huella y su rastro a modo de identidad digital. La cultura de la participación implica relaciones que permiten compartir, resolver problemas, desarrollar tareas, construir conjuntamente conocimiento y desarrollar canales de comunicación dinámicos. Esta cultura de la participación aplicada a contextos educativos permite crear oportunidades de aprendizaje y diversificar en los social media un mayor poder de la ciudadanía como usuaria digital.

\section{Palabras clave}

Cultura digital; Identidad digital; Posicionamiento; Privacidad; Reputación.

\section{Sumario}

1. Introducción. 2. Cultura de la participación y roles de la ciudadanía. 3. Identidad digital. 4. Conclusiones. 5. Referencias bibliográficas. actor, with the capacity to create within a digital space one's own information and communicative style. This digital citizenship in virtual scenarios leaves traces and footprints in the form of a digital identity. The culture of participation involves relations that allow sharing, problem solving, the performance of tasks, the construction of collaborative knowledge, and the development of dynamic channels of communication. This culture of participation applied in educative contexts allows the creation of learning opportunities and the diversification of social media as an increased power toward citizenship for digital users.

\section{Keywords}

Digital culture; Digital identity; Privacy, Positioning; Reputation.

\section{Summary}

1. In the gothic 9th century, justified: 1. Introduction. 2. Culture of Participation and the role of the citizen. 3. Digital Identity. 4. Conclusions. 5. Bibliographic References.

\section{Introducción}

La cultura de la participación es una de las características de las primeras décadas del siglo XXI. La cultura de la participación es aquella que no tiene barreras para la expresión ciudadana, que apoya la creatividad y la puesta en común de creaciones propias y colectivas. Los individuos creen en la importancia que tienen sus contribuciones y sienten una conexión entre lo que dicen los demás y sus propias aportaciones.

Participar implica formar parte, colaborar con los demás y conformar el grupo de consenso para conseguir metas comunes. En realidad, la participación es un derecho de la ciudadanía y así debe ejercerse de forma libre, crítica y responsable en todos los sectores de la vida. La participación, como derecho fundamental reconocido en cualquier sistema democrático, requiere de un desarrollo normativo y de políticas que apuesten por el derecho de la ciudadanía. En caso contrario, la participación ciudadana no se dará en la medida deseable puesto que la propia estructura social, jerarquizada y burocratizada, permitiría escaso margen para ello. 


\section{Cultura de la participación y roles de la ciudadanía}

El camino para construir una cultura de la participación es largo, muy complejo y laborioso. Una de las claves se encuentra en el modelo de comunicación que se establezca para dicha participación. Una comunicación horizontal es la base para establecer una relación colaborativa basada en el consenso y la confianza mutua. Este binomio consenso - confianza puede ser la clave del éxito para conseguir una verdadera cultura de la participación.

En una sociedad digital que pretende ser verdaderamente democrática, la transparencia y la participación en las decisiones de la propia sociedad es un aspecto fundamental a tener en cuenta. La participación no es un fin en sí misma sino que más bien es un medio. La participación es la que permite tomar parte e intervenir en la vida social y se manifiesta como herramienta al servicio de la ciudadanía y no debe quedar solamente en un plano ideológico de un discurso banal

Por tanto, la participación es una forma de actuación activamente democrática y efectiva que afecta positivamente a la vida pública. La participación es una responsabilidad que supone un compromiso, un compromiso solidario y crítico con el conjunto de la sociedad, a la hora de tomar decisiones y actuaciones colectivas. A todas luces, en y con la participación, el individuo se compromete y cree en la justicia social y en el respeto a los demás con la idea de transformar la sociedad en la que vive en un sitio mejor, acorde con los derechos humanos en el contexto digital que se está construyendo y creando día a día .

Asociado a esta concepción del concepto de participación se encuentra otro término fundamental, la interactividad. La diferencias entre ellos es que mientras la interactividad es una propiedad que proporciona la tecnología, la participación es una propiedad que proporciona la cultura. Ambas, interactividad y participación, son términos relacionados y dependen de la formación de las personas que las lleven a cabo.

Junto a los conceptos de interactividad y participación surge el término de convergencia, que cambia radicalmente la idiosincrasia de nuestra época. La convergencia digital que se está produciendo en estos momentos conlleva tanto cambios tecnológicos como cambios comunicativos, culturales y sociales. No debemos quedarnos en la idea de que la convergencia digital implica sólo grandes avances de los soportes tecnológicos. En paralelo a los cambios derivados de que desde cualquier soporte móvil podamos desarrollar cualquier acción en el ciberespacio, aparecen nuevas manifestaciones comunicativas y culturales asociadas. En primer lugar, Osuna y Busón (2007) afirman que estamos condicionados por el hecho de que las tecnologías acompañan constantemente a los individuos las 24 horas de los 365 días del año. Esto hace que las formas de comunicarnos cambien y se caractericen cada vez más por la inmediatez de respuesta. Por otro lado, el acceso a la información en cualquier lugar y en cualquier momento permite que asistamos a una época en la que la ciudadanía tiene mayor poder de participación en la cultura de su época como no la ha tenido en ningún otro momento en la historia de la 
humanidad. La ciudad comienza a percibir su propio potencial comunicativo a escala local y global, a tal punto que están creando redes informativas paralelas $y$, muchas veces independientes, a las que establecen los grandes medios de comunicación y las industrias de la cultura. Las narraciones ya no están sólo en manos de las grandes empresas y de las grandes corporaciones, sino que también depende de los propios ciudadanos y ciudadanas, que tienen la posibilidad de tomar el control de los medios sociales y contar sus propias teorías de forma diferente y en sus contextos correspondientes. Jenkins (2008) afirma que:

"el término "cultura participativa" contrasta con opciones más antiguas del espectador mediático pasivo. Más que hablar de productores y consumidores mediáticos como si desempeñasen roles separados, podríamos verlos hoy como participantes que interactúan conforme a un nuevo conjunto de reglas que ninguno de nosotros comprende del todo. No todos los participantes son creados iguales. Las corporaciones, e incluso los individuos dentro de los medios corporativos, ejercen todavía un poder superior al de cualquier consumidor individual o incluso al del conjunto de consumidores. $Y$ unos consumidores poseen mayores capacidades que otros para participar en esta cultura emergente" (p. 15).

Aunque los soportes tecnológicos estén muy desarrollados, por sí mismos no son capaces de crear el fenómeno de la convergencia de medios. Es necesario que las personas se apropien de dicha tecnología e interactúen socialmente a través de ella, por lo que podemos afirmar que la convergencia se produce en el cerebro de la ciudadanía en el momento que actúan colectivamente.

Cuando Pierre Lévy (2004) alude al concepto de inteligencia colectiva, nos manifiesta que ninguna persona es capaz de saberlo todo, sino que cada uno sabe algo y, si juntamos lo de cada cual y lo combinamos, podemos llegar a crear un intelecto común que se convierte en una gran alternativa de poder en los nuevos escenarios de participación. La inteligencia colectiva que propone Lévy debe entenderse como una fuente alternativa de poder mediático. La inteligencia colectiva es mucho más que la suma de individualidades.

La inteligencia colectiva no aparece porque haya una configuración tecnológica $u$ otra, sino más bien es algo que se conforma en la medida en que actuemos conjuntamente. La cultura de la participación engloba nuevas formas de cocreación en el ciberespacio, de ahí que Berners Lee, citado por Osuna (2010), al referirse el concepto de intercreatividad dice que el:

"término alude a la capacidad de los individuos de expresar y crear sus ideas, conocimientos, etc. de una forma original e inédita a través de los entornos digitales y generando conocimiento colectivo. Este autor afirma que la intercreatividad facilita los mecanismos para que los 
individuos interactúen creando en comunidad. La convergencia digital no sólo se produce en nuestras cabezas, sino también en nuestras interacciones sociales dentro del ciberespacio" (p. 140 - 141).

En este sentido, Lessig da un paso más cuando nos habla de la cultura de la remezcla (remix culture) definiéndola como el concepto por el que la sociedad usa todo tipo de elementos existentes para crear cosas nuevas. Este autor afirma que la remezcla mediática "tiene éxito cuando muestra algo nuevo a los otros y fracasa cuando es manida y poco original. Como un buen ensayo o un buen chiste, una remezcla se inspira en la obra de los otros para crear una nueva" (Lessig, 2013: 117).

Y la participación intercreativa a escala individual y colectiva se está dando a dos niveles, a partir de la producción de obras originales y la producción remix. Estas producciones remix son una reescritura no sólo de una obra en particular sino que suele implicar interpretaciones de la cultura, la política, la economía, etc. Estas interacciones comunicativas entre los usuarios rompen con una tradición funcionalista que los calificaba como audiencia.

En el contexto digital el concepto de audiencia como meramente receptora se ve sustancialmente modificado. Este término queda limitado o circunscrito a medios analógicos. Emergen individuos participativos e implicados con la información que circula en el ciberespacio con una interacción constante con sus iguales, a modo de "perceptores participantes" (Marta Lazo, 2008). Manuel Castells (1998) como decimos más arriba clasificaba a los individuos como "interactuantes" e "interactuados" para hacer constar los nuevos roles que se asumen en la red. Es decir, "aquellos capaces de seleccionar sus circuitos de comunicación multidireccionales y aquellos a los que se les proporcione un número limitado de opciones preempaquetadas" (Castells, 1998: 404) respectivamente; enfoque distinto a las categorías que $G^{a}$ Canclini enunciara en 1995 de "consumidores pasivos" y "consumidores activos". En este ecosistema comunicacional de la participación adquieren especial relieve Pisani y Piotet (2009) cuando abogan por el término "webactores" en escenarios digitales, definiéndolos como individuos con la capacidad de producir, actuar y modificar la Web. En este sentido se habla de nuevas interacciones: de la recepción al empoderamiento, como apropiación de contenidos, que es un proceso autónomo en su individualidad y procomún en su colectividad, como indican Marta y Gabelas (2013).

Internet no desechará el viejo orden analógico ni su época de la web 1.0, sino que la renovación que produce el desarrollo de las redes sociales va a crear un nuevo paradigma digital participativo. Para Jenkins, "los viejos medios nunca mueren, y ni siquiera se desvanecen. Lo que mueren son simplemente las herramientas que utilizamos para acceder al contenido de los medios [...] Las tecnologías de distribución quedan obsoletas y son reemplazadas; por otra parte, los medios evolucionan" (Jenkins, 2008: 24). Desde este punto de vista, la televisión no hizo desaparecer a la radio ni el 
cine al teatro. Cabe suponer que el nuevo escenario digital participativo ocupará su espacio con sus propias peculiaridades y normas. En este sentido, los viejos medios nunca mueren, lo que muere en todo caso son las herramientas que utilizamos para acceder a sus contenidos. Morirán las tecnologías de distribución, pero los medios evolucionarán y se afianzarán.

Ya en 2005, O`Reilly enunció el principio de la arquitectura de la participación, que es una de las mayores cualidades de la Web 2.0. Ya que provee de muchos instrumentos tecnológicos facilitadores de la colaboración y relaciones sociales de los sujetos de la red. Esta arquitectura de la participación promueve una nueva gestión del conocimiento y $O$ 'Reilly afirmaba que la Web 2.0 que se está gestando no es una mera tecnología, sino una actitud de la ciudadanía ante nuevos escenarios digitales con una ética de colaboración implícita.

Los cambios mediáticos, por tanto, no se pueden reducir en función de los cambios tecnológicos. Con la convergencia digital tenemos la tecnología que hace posible la comunicación, por un lado, y las prácticas sociales y culturales desarrolladas a través de esa tecnología, por otro. Precisamente por los hábitos y las prácticas colaborativas que lleva a cabo la ciudadanía se está realizando una dinámica interna de reapropiación y resignificación de la información, del conocimiento.

En esta nueva concepción de uso, los social media están siendo los vehículos por los que se ha propiciado la cultura de la participación y es donde se observa la gran mayoría de las nuevas tendencias infocomunicativas. Los movimientos como giving\&partipate participar y compartir y las nuevas figuras como el community manager o el curator se vuelven en máximas para la participación.

La cultura de la participación en los social media implica los siguientes cambios:

- menos barreras para la expresión ciudadana,

- que la Web 2.0, en general, y las redes sociales, en particular, supongan el soporte para la creatividad y para compartir conocimiento,

- mayor valor para la experiencia y

- valorar las opiniones y recomendaciones de los iguales como forma coconstruir una nueva sociedad más justa y democrática.

Estos preceptos se están llevando a cabo de forma espontánea y gregaria, se están conformando a través de la propia práctica. Las formas de asociación de este nuevo ecosistema son similares a las primeras formas de reagrupamiento de los seres humanos, motivados por intereses, gustos, similitudes, idioma,... Es una práctica comunicativa natural que va creciendo a medida que se lleva a cabo y que requiere de un proceso de reflexión, de análisis y de estudio de lo que acontece en la propia red. En definitiva, un proceso de alfabetización digital ayudaría a la participación.

Esta alfabetización digital para la participación implica la acción colectiva y la creación de estructuras que faciliten la comunicación, donde los espacios para el debate y la toma de decisiones tendrían un papel 
protagonista. La participación lleva implícito una movilización social que no se puede hacer sólo con la intención de querer participar, sino que hay que saber participar. Esto quiere decir que antes de participar hay que saber para qué, cómo, de qué forma,... se puede participar. La participación requiere de una formación que permita adquirir los conocimientos y habilidades necesarias para comunicarse y relacionarse con otras personas con un fin colectivo. Desde la educación formal, no formal e informal se deben tener actuaciones al respecto. En realidad es una educación en valores para la participación que debe estar presente en todos los ámbitos sociales.

La cultura participativa debe formar parte del currículo oculto de las instituciones educativas. Quienes se formen en ellas deben estar en contacto con este currículo para tener un acceso igualitario a las oportunidades sociales y a las experiencias que se planteen para la capacitación y el conocimiento.

Henry Jenkins (2008) habla de la educación del siglo XXI como aquella que debe atender las habilidades sociales necesarias para la cultura de la participación. Esto no quiere decir que las habilidades heredadas de la cultura de la imprenta tengan que ser desechadas, sino que deberían ser ampliadas con las competencias que requiere la cultura digital. La educación se enfrenta a enormes problemas para educar en la participación, entre los que se encuentran los rápidos cambios y las transformaciones complejas y difíciles de analizar y de comprender. La escuela del siglo XXI tiene nuevos retos y necesita nuevas respuestas. En la actualidad,

"la gente está aprendiendo a participar en estas culturas del conocimiento al margen de cualquier entorno educativo formal. Buena parte del aprendizaje tiene lugar en los espacios de afinidad que surgen en la cultura popular. El nacimiento de estas culturas del conocimiento refleja en parte las exigencias que estos textos plantean a los consumidores (la complejidad del entretenimiento transmediático, por ejemplo), pero también refleja las exigencias que los consumidores plantean a los medios (el ansia de complejidad, la necesidad de comunidad, el deseo de reescribir historias fundamentales. Muchas escuelas permanecen abiertamente hostiles a esta clase de experiencias" (p. 256)

Se presenta un nuevo escenario global donde surgen nuevas formas de expresión cultural, nuevas escalas de valores y nuevas estructuras de la sociedad. Se hace necesario desarrollar actuaciones comunicativas que pongan en contacto dinámicas de participación locales y cercanas con otras dinámicas globales y lejanas que pueden ser de dos tipos:

- las que se producen de forma natural

- las buscadas y que responden a una estrategia consciente, a un proceso de conexión deliberado para la discusión creativa y reflexiva. 
En este último caso, la participación requiere de la construcción consciente de la identidad digital.

\section{Identidad digital}

Estudios como los de Livingstone (2004), Perkel (2006) y Zhao (2008) nos dan idea de la importancia de la identidad digital. Identidad 2.0 o identidad digital es todo lo que manifestamos en el ciberespacio e incluye tanto nuestras actuaciones como la forma en la que nos perciben los demás en la red. La identidad se crea conforme vamos actuando dentro del espacio digital. Todas nuestras acciones constituyen parte de nuestra identidad, así como nuestras omisiones o todo lo que dejamos de hacer. Cuando estamos conformando nuestra identidad digital es muy importante saber que ésta es lo que somos para los demás, es decir, lo que el ciberespacio dice que somos a los demás. Todos los datos como imágenes que subimos, comentarios que escribimos, clics sobre los enlaces por donde navegamos, contexto donde interactuamos y el lugar donde estén accesibles nuestros datos nos identifican y conforman nuestro perfil digital. La gestión de la identidad digital es una nueva habilidad que debe desarrollar la ciudadanía dentro de sus competencias personales. Las habilidades informacionales, comunicativas y mediáticas se aplican a todos los niveles, cuando sentimos la necesidad de buscar información y cuando nos queremos comunicar con alguien, teniendo siempre en cuenta el uso ético y legal que implica una correcta cultura de la participación.

Es necesario conseguir una presencia en Internet gestionada eficazmente en función de la evolución de la cultura digital. Por ejemplo, lo que en la modernidad podría tener lugar en una conversación privada, ahora se lleva a cabo con mensajes públicos en un blog o en las redes sociales. Aparece una nueva concepción pública de comunicación a través de microblogs, con un número más limitado de caracteres que los blogs, donde el diálogo fluye en pequeños fragmentos y se tiene la posibilidad de seguir a otras personas para conocer qué hacen y qué escriben. Personalidades representativas de los distintos campos del conocimiento son seguidas por millones de individuos para conocer en qué trabajan o qué piensas sobre los temas de actualidad.

En muchas ocasiones los individuos no tienen solo una sola identidad digital sino que pueden crearse varias identidades diferentes en Internet. A su vez, la identidad que una persona se crea puede coincidir o no con la identidad analógica, es decir, con las características de esa persona en el espacio físico. Cualquier persona inmersa en el espacio digital, en general, gestiona muchísimos datos que son difícilmente calculables y que es, la mayoría de las veces, incontrolable por la misma persona, por lo que se exige la necesidad de nuevas alfabetizaciones para capacitar al individuo en las nuevas demandas sociales. Uno de los requisitos necesarios a la hora de gestionar la identidad digital es que hay que estar inmersos en la propia cultura digital, participar de ella, y las personas que no tienen facilidad de acceso al ciberespacio siempre van a encontrar una brecha digital en 
este sentido. De aquí que podamos afirmar que el ciberespacio va a convertirse en un medio con bastante riesgo de exclusión social.

Una identidad digital bien gestionada y acorde con la identidad analógica repercute en una vida más activa en todos los ámbitos, además de consolidar un entramado de actuación más coherente. De hecho, necesitamos construir la identidad digital ligada al desarrollo de habilidades informacionales y comunicativas a través de una actitud activa en Internet, abierta y colaborativa. En la identidad digital convergen muchos aspectos de carácter sociológico, cultural y psicológico. Wood y Smith definen la identidad digital como una construcción compleja, personal y social, teniendo en cuenta que en esa construcción se incluye los matices de cómo queremos que los demás no perciban y cómo, en realidad, nos perciben. La identidad digital repercute en la identidad física o analógica y viceversa.

La identidad digital no se gestiona de forma instrumental, sino que es un conjunto de habilidades que deben aprenderse a lo largo de la vida para llevar a cabo cuando el individuo esté inmerso en la cultura de la sociedad red. Para gestionar la identidad digital se hace necesario gestionar los siguientes elementos: visibilidad, posicionamiento web, reputación y privacidad en Internet.

El concepto de visibilidad está muy relacionado con la actividad que genera un individuo en la red. La puede construir la propia persona a partir de las publicaciones o mensajes en redes sociales, videos subidos, blogs, etc. pero también esta visibilidad puede venir dada a través de referencias o comentarios de otras personas. Es frecuente que al instalar un app en nuestros dispositivos móviles se nos pregunte, por ejemplo, si queremos permitir que la propia aplicación localice nuestra ubicación. La visibilidad aumenta cuando los propios sujetos envían noticias a las redes sociales o a otras herramientas, de tal manera que la información se distribuye de forma muy eficaz. De hecho, la visibilidad de una persona se puede medir y las redes sociales son un ejemplo claro. Se mide el número de contactos, el número de seguidores, los contenidos replicados. El tráfico de contenidos que se mueve desde unos espacios a otros dentro de Internet constituye un indicador de visibilidad cuantificable.

El posicionamiento web u optimización de motores de búsqueda, también llamado por su nombre en inglés, SEO (Search Engine Optimization), está íntimamente relacionado con el concepto de visibilidad. Un buen posicionamiento web implica un aumento de la visibilidad en el ciberespacio. Se trata de un concepto que va más allá de incorporar palabras claves o etiquetas meta tag en los sitios web para que los buscadores las localicen, pues existen tantas etiquetas que dejan de ser relevantes. Además de estas palabras claves, el posicionamiento en la Web 2.0 se basa en la cantidad y fuerza de los sitios para ser visitados por los individuos conectados a Internet que navegan aleatoriamente. En este sentido, con el aumento de las redes sociales y los blogs, principalmente, el tráfico de datos que exista respecto a una determinada identidad digital es lo que la va a posicionarla mejor o peor en el ciberespacio. Se puede 
optimizar el posicionamiento web de cualquier persona de dos formas, incorporando contenidos accesibles de calidad y consiguiendo notoriedad y seguimiento de dichos contenidos.

La reputación del sujeto o reputación que define la identidad digital de una persona no recae en la propia persona, sino en las opiniones que otras personas tienen del sujeto. Esto no quiere decir que el propio individuo no pueda tener parte activa en su reputación. Solove (2007) afirma que la reputación es un elemento clave para la identidad de los individuos, ya que define cómo interactúa con los demás. La reputación se va generando en virtud a quien habla de qué en este sitio y de qué forma, por tanto, si esa opinión se muestra en un sitio muy visible se difundirá de manera muy rápida. La red social Linkedin, por ejemplo, es un espacio que gestiona muy bien la reputación en Internet. Cada usuario o usuaria crea un perfil y se puede comunicar con otras personas de su campo profesional. Se puede recomendar a una persona o añadir un comentario con una breve explicación sobre las cualidades de cualquier profesional y eso va a ser recibido por quien esté conectado en su ámbito laboral.

La privacidad es otro de los elementos que conforman la identidad digital y cualquier persona debería tener la capacidad suficiente de conocer los elementos mínimos de privacidad que debe mantener en Internet. El tema de la privacidad es un aspecto clave en la sociedad digital. Cuando una persona instala cualquier aplicación en sus dispositivos electrónicos está firmando un contrato que le permite el uso de dicha aplicación. Al aceptar las condiciones está dejando las manos libres a la aplicación para que haga uso de la información que contiene de cada uno de sus usuarios y tiene la potestad de poderla ofrecer a terceros. Los datos como fotografías, direcciones de correo electrónico, currículo profesional, gustos, etc. se dejan a menudo al alcance de perfectos desconocidos que son los que gestionan todos los datos que se reciben en las redes sociales. Cuando una persona introduce sus datos, en realidad está cediendo toda su información y, en muchos casos, aunque el individuo se dé de baja en ese espacio digital estos datos van a quedar visibles. En este sentido, la alfabetización digital tiene como objetivo formar a los individuos para que sean conscientes de estos aspectos y adquieran las competencias necesarias sobre lo que pueden o no pueden compartir. Todo lo que se publica en Internet queda para la posteridad, con lo cual es difícil dar marcha atrás. En la vida física, al cabo de muchos años, en la memoria colectiva se van perdiendo muchos hechos o acontecimientos, sin embargo, en la vida digital los datos nunca se pierden, siempre están ahí, siempre están presentes.

En realidad, la construcción de la identidad en el ciberespacio es un aprendizaje tanto personal como profesional dentro de la cultura digital en la que estamos inmersos actualmente. Podemos hablar de una identidad híbrida entre la identidad digital y la identidad analógica de los individuos. 


\section{Conclusión}

En esta última década hemos asistido a la eclosión espontánea de la cultura de la participación a escala global. Estos primeros años han servido para intuir el potencial de la inteligencia colectiva y el poder de la comunicación entre iguales donde se hacen públicos desde aspectos cotidianos hasta manifestaciones políticas, educativas o sociales.

La participación puede ser espontánea como una marea de ideas ricas en movimiento constante y sin objetivos claros o también puede tener una identidad propia, grados de visibilidad intencionados, reputación y posicionarse en la web. En este último caso, la participación ha de constituirse en una estrategia cultural de los diferentes grupos sociales para reinventar el poder ciudadano digital.

\section{Referencias bibliográficas}

[1] APARICl, R. (coord.) (2010). Conectados en el ciberespacio. Madrid: UNED.

[2] BERNERS-LEE, T. (2008). Tejiendo la Red. El inventor del World Wide Web nos descubre su origen. Madrid: Siglo Veintiuno de España Editores.

[3] CASTELLS, M. (1998). La era de la información: Economía, sociedad y cultura. Vol. 1 La sociedad red. Madrid: Alianza Editorial.

[4] GARCÍA CANCLINI, N. (1995). Consumidores y ciudadanos. Conflictos multiculturales de la globalización. México: Grijalbo.

[5] JENKINS, H. (2008). Convergence Culture. La cultura de la convergencia de los medios de comunicación. Barcelona: Ediciones Paidós Ibérica S.A.

[6] LESSIG, L. (2013). Remix. Cultura de la remezcla y derechos de autor en el entorno digital. Barcelona: Icaria Editorial S.A.

[7] LÉVY, P. (2004). Inteligencia Colectiva. Por una antropología del ciberespacio. Washintong, DC.: Organización Panamericana de la Salud. Unidad de Promoción y Desarrollo de la Investigación y el Centro Latinoamericano y del Caribe de Información en Ciencias de la Salud.

[8] LÉVY, P. (2007). Cibercultura. La cultura de la sociedad digital. Barcelona. Anthropos: Editorial del Hombre.

[9] LIVINGSTONE, S. (2004). Media literacy and the challenge of new information and communication Technologies. The Communication Review, no. 7, pp. 3-14 Recuperado el día 20 de octubre de 2013 de http://eprints.Ise.ac.uk/1017/1/MEDIALITERACY.pdf

[10] MARTA-LAZO, C. Y GABELAS BARROSO, J.A. (2013). Nuevas interacciones de la competencia digital: de la recepción al empoderamiento. En Morales, S y Loyola, M. I. (compiladoras). Nuevas perspectivas de los estudios de la comunicación. La apropiación tecnomediática (pp. 65-78). Buenos Aires: Imago Mundi. 
[11] MARTA-LAZO, C. (2008). El proceso de recepción televisiva como interacción de contextos. Comunicar, pp. 35-40.

[12] O'REILLY, T. (2005). What is Web 2.0? Design Patterns and Business Models for the Next Generation of Software. O'Reilly Network. Recuperado el día 20 de octubre de 2013 de http://www.oreillynet.com/pub/a/oreilly/tim/news/2005/09/30/what-is-web20.html

[13] OSUNA, S. y BUSÓN, C. (2007). Convergencia de Medios. La Integración Tecnológica en la Era Digital. Barcelona: Icaria Editorial S.A.

[14] OSUNA, S. (2010). Interactuantes e interactuados. En Aparici, R. (coord.), Conectados en el ciberespacio. Madrid: UNED.

[15] PERKEL, D. (2006). Copy and paste literacy: literacy practices in the production of a MySpace profile. Informal learning and digital. Recuperado el día 20 de octubre de 2013 de http://people.ischool.berkeley.edu/ dperkel/media/dperkel_literacymyspa ce.pdf

[16] PISANI, F. y PIOTET, D. (2009). La Alquimia de las Multitudes. Cómo la Web está Cambiando el Mundo. Barcelona: Ediciones Paidós Ibérica S.A.

[17] SOLOVE, D. J. (2007). Rumor and reputation in a digital world. En: Solove, Daniel J. The future of reputation: gossip, rumor, and privacy on the Internet. New Haven: Yale University Press.

[18] WOOD, Andrew F. y SMITH, Matthew J. (2005). Forming online identities. En: Wood, Andrew F. y Smith, Matthew J. Online communication: linking technology, idenity, and culture. Mahwah, N. J.: L. Erlbaum.

[19] ZHAO et al. (2008). Identity construction on Facebook: digital empowerment in anchored relationships. Computers in human behavior, vol. 24, pp. 1816-1836. Recuperado el día 20 de octubre de 2013 de http://astro.temple.edu/ bzhao001//dentity\%20Construction\%20on\%20Fac ebook.pdf 